\title{
PROVISIONAL BEST PRACTICES GUIDELINES FOR THE EVALUATION OF BULBAR DYSFUNCTION IN AMYOTROPHIC LATERAL SCLEROSIS
}

GARY L. PATTEE, MD, ${ }^{1}$ EMILY K. PLOWMAN, PHD/CCC-SLP $\mathbb{D}^{2}{ }^{2}$ KENDREA L. (FOCHT) GARAND, PHD, CSCD/CCC-SLP, ${ }^{3}$ JOHN COSTELLO, MA/CCC-SLP, ${ }^{4}$ BENJAMIN RIX BROOKS, MD, ${ }^{5}$ JAMES D. BERRY, MD, MPH, ${ }^{6}$ RICHARD A. SMITH, MD, ${ }^{7}$ NAZEM ATASSI, MD, ${ }^{6}$ JENNIFER L. CHAPIN, MA/CCC-SLP, ${ }^{2}$ YANA YUNUSOVA, PHD/CCC-SLP, ${ }^{8}$ COURTNEY E. MCILDUFF, MD, ${ }^{9}$ EUFROSINA YOUNG, MD, ${ }^{10}$ ERIC A. MACKLIN, PHD, ${ }^{11}$ EDUARDO R. LOCATELLI, MD, MPH, ${ }^{12}$ VINCENZO SILANI, MD, ${ }^{13}$ DARAGH HEITZMAN, MD, ${ }^{14}$ JAMES WYMER, MD, PHD, ${ }^{15}$ STEPHEN A. GOUTMAN, MD, MS $\mathbb{D}^{16},{ }^{16}$ DEBORAH F. GELINAS, MD, ${ }^{17}$ BRIDGET PERRY, PHD/CCC-SLP, MS, ${ }^{4}$ PAIGE NALIPINSKI, MA/CCC-SLP, ${ }^{18}$ KAILA STIPANCIC, MA/CCC-SLP, ${ }^{4}$ MEGHAN O'BRIEN, MS/CCC-SLP, ${ }^{4}$ STACEY L. SULLIVAN, MS/CCC-SLP, ${ }^{18}$ ERIK P. PIORO, MD, PHD, ${ }^{19}$ GISELLA GARGIULO, MD, PHD, ${ }^{20}$ JORDAN R. GREEN, PHD/CCC-SLP, ${ }^{4}$ and CONTRIBUTING MEMBERS OF THE NEALS BULBAR SUBCOMMITTEE

${ }^{1}$ Neurology Associates P.C, Lincoln, NE, U.S.A.

${ }^{2}$ Department of Speech, Language and Hearing Sciences, University of Florida, Gainesville, FL, U.S.A.

${ }^{3}$ Department of Speech Pathology and Audiology, University of South Alabama, Mobile, AL, U.S.A.

${ }^{4}$ Speech and Feeding Disorders Lab, MGH Institute of Health Professions, Charlestown, MA, U.S.A., ALS Augmentative Communication Program, Boston Children's Hospital, Boston, MA, U.S.A.

${ }^{5}$ Carolinas Medical Center, Charlotte, NC, U.S.A.

${ }^{6}$ MGH Department of Neurology, Boston, MA, U.S.A.

${ }^{7}$ Center for Neurologic Study, San Diego, CA, U.S.A.

${ }^{8}$ Department of Speech-Language Pathology, University of Toronto, Toronto, ON, CANADA

${ }^{9}$ Beth Israel Deaconess Medical Center, Boston, MA, U.S.A.

${ }^{10}$ State University of New York, Department of Neurology, Syracuse, NY, U.S.A.

${ }^{11}$ MGH Biostatistics Center, Boston, MA, U.S.A.

${ }^{12}$ Holy Cross Hospital, Department of Neurology, Fort Lauderdale, FL, U.S.A.

${ }^{13}$ Istituto Auxologico Italiano, IRCCS, Department of Neurology and Laboratory of Neuroscience, Department of Pathophysiology and Transplantation, "Dino Ferrari" Center, Università degli studi di Milano, Milan 20122, Italy

${ }^{14}$ Texas Neurology, P.A, Dallas, TX, U.S.A.

${ }^{15}$ University of Florida, Rehabilitation Science, Gainesville, FL, U.S.A.

${ }^{16}$ University of Michigan, Department of Neurology, Ann Arbor, MI, U.S.A.

${ }^{17}$ UNC Health Care, Chapel Hill, NC, U.S.A.

${ }^{18}$ MGH Speech Language Pathology, Boston, MA, U.S.A.

${ }^{19}$ Cleveland Clinic, Department of Neurology, Cleveland, OH, U.S.A.

${ }^{20}$ National Scientific and Technical Research Council, Buenos Aires, Argentina

Accepted 2 January 2019

ABSTRACT: Introduction: Universally established comprehensive clinical bulbar scales objectively assessing disease progression in amyotrophic lateral sclerosis (ALS) are currently lacking. The goal of this working group project is to design a best practice set of provisional bulbar ALS guidelines, available for immediate implementation within all ALS clinics. Methods: ALS specialists across multiple related disciplines participated in a series of clinical bulbar symposia, intending to identify and summarize the currently accepted best practices for the assessment and management of bulbar dysfunction in ALS Results: Summary group recommendations for individual speech, Augmentative and Alternative Communication (AAC), and swallowing sections were achieved, focusing on the optimal proposed level of care within each domain. Discussion: We have identified specific clinical

\footnotetext{
Abbreviations: AAC, Alternative and Augmentative Communication; ALS, amyotrophic lateral sclerosis; ALS-FRS-R, ALS functional rating scalerevised; CNS-BFS, Center for Neurologic Study Bulbar Function Scale; CPF, cough peak flow; FVC, forced vital capacity; NEALS, Northeast ALS Consortium; PBA, pseudobulbar affect; SLP, speech-language pathologist; SVC, slow vital capacity; VFSS, Videofluoroscopic Swallow Study. Key words: Bulbar; Speech; Swallowing; Guidelines; AAC

Funding: Cytokinetics Pharmaceuticals, Center for Neurologic Study (CNS) Conflicts of Interest: The authors declare no financial conflicts of interest for the work presented in this article.
}

Correspondence to: Gary L. Pattee, M.D. 2631 South 70 Street Lincoln, NE 68506; glpattee@gmail.com

() 2019 Wiley Periodicals, Inc

Published online 5 February 2019 in Wiley Online Library

(wileyonlinelibrary.com). DOI 10.1002/mus.26408 recommendations for each of the 3 domains of bulbar functioning, available for incorporation within all ALS clinics. Future directions will be to establish a formal set of bulbar guidelines through a methodological and evidence-based approach.

Muscle Nerve 59:531-536, 2019

Bulbar dysfunction in amyotrophic lateral sclerosis (ALS) significantly contributes to reductions in quality of life, social withdrawal, malnutrition, dehydration, aspiration pneumonia, and early mortality. A globally recognized working metric, incorporating reliable clinical assessment scales to monitor bulbar disease progression in ALS, has yet to be achieved. Previous attempts aimed at achieving this metric have fallen short of a satisfactory and comprehensive protocol. ${ }^{1-5}$ The Northeast ALS (NEALS) bulbar subcommittee has recently completed a bulbar practice survey regarding current practice patterns within participating sites, which identified significant inconsistencies involving the assessment and management of bulbar dysfunction in ALS. ${ }^{6}$ These survey results revealed an urgent need to design and incorporate a best practice set of provisional guidelines, intended to comprehensively assess and monitor bulbar dysfunction across clinical sites. In an attempt to 
address this need, the NEALS bulbar subcommittee recently convened to generate a provisional working group set of best practice guidelines, as established through expert standard of care consensus. Our expected goals are to: (1) Standardize bulbar data collection across all sites, which will assist in the acquisition of patient data; (2) Develop guidelines for the timing of referrals and follow-up evaluations of speech, augmentative and alternative communication (AAC), and swallowing; and (3) Propose common data elements for standardized data collection of speech, AAC, and swallowing assessments. The temporal feasibility of implementing these guidelines within the clinic setting was a critical concern, emphasizing the required time and ease of administration, given the amount of clinical testing ALS patients routinely undergo from the multiple health related disciplines during each clinic visit. A pragmatic approach was, therefore, underscored.

\section{METHODS}

Several designated ALS bulbar symposia recently convened to establish consensus summary recommendations for the assessment of bulbar dysfunction, specifically related to the domains of speech, AAC, and swallowing. In attendance were ALS specialists across multiple disciplines, including clinical neurology, biostatistics, and speech-language pathology (SLP) representing the United States, Canada, and Italy, thereby providing an expansive range of bulbar expertise from an experienced clinical perspective. This consensus group worked to establish provisional guidelines, focusing on speech and swallowing impairments. The focus of the speech group was to (1) provide guidance concerning the timing of patient referrals for a speech and AAC evaluation and (2) propose common data elements of a clinical speech exam that are feasible to administer in a busy ALS clinical setting. The working goal of the swallowing group was to develop a pragmatic, triage-based clinical pathway to guide screening, assessment, and management of dysphagia in patients with ALS. These symposium sessions were organized into separate speech, AAC and swallowing sections, resulting in working group summary guidelines within each section that ultimately underwent a comprehensive review by the entire committee.

\section{RESULTS}

Speech Section: Clinical Speech Evaluation. The speech section focused on the establishment of important supplementary metrics of related systems and functions, with subsequent system and subsystem analysis: forced vital capacity/slow vital capacity (FVC/SVC), ALS Functional Rating Scale Revised (ALSFRS-R), pseudobulbar affect (PBA), dysphagia, medications, and cognition.

Guidance regarding Speech referrals. The speech section recommended SLP speech evaluations at the initial clinic visit and recommended that all clinic visits should gather routine clinical information as listed in Figure 1. Follow-up speech assessments are suggested as an integral component of each ALS clinic visit, although frequency and visit duration may vary depending on multiple factors including patient needs, SLP availability, physician recommendations, and clinic resources. All patients who present with an oral motor exam atypical for bulbar ALS (i.e., asymmetrical tongue or pharyngeal weakness, oral/throat pain, raspy voice, or swallow difficulties preceding speech impairment) should undergo an otolaryngology evaluation to rule out alternative causes.

Common data elements of clinical speech examination. The common elements of the speech examination are listed in Figure 1. The Center for Neurologic StudyBulbar Function Scale (CNS-BFS), a recently validated patient reported outcome measure ${ }^{7}$ that has demonstrated clinical utility, ${ }^{8}$ may also include a family member or caregiver for guidance to more completely assess the communication status of the patient. The speech assessment should include a spontaneous speech sample and the reading of a short paragraph, such as the Bamboo $^{9}$ or Rainbow Passage ${ }^{10}$ (only for fluent English speakers and readers; in non-English speakers/readers another short paragraph should be selected based upon the patient's native language). These samples will allow clinician-based ratings of dysarthria severity $(0=$ normal, 4 = severe), speaking rate (words per minute), and involvement of 1 or more of the 4 speech subsystems (respiratory, phonatory, articulatory, and resonatory). Clinician based ratings of speech should be supplemented with objective testing of the involved individual speech subsystems (Fig. 2). The estimated time necessary to perform the entire speech assessment is 8 to $10 \mathrm{~min}$.

AAC Section: Clinical Communication Evaluation. The AAC section defined the scope of augmentative communication options, identifying the optimal timing of an evaluation and stressed a proactive approach. It also focused upon minimizing the patient energy expenditure while maximizing their collaborative engagement. Guidance on when to refer for speech and AAC evaluations, and frequency of follow-up, was established. Understanding that speech can rapidly deteriorate, patient education with AAC exposure and training is critical during the early stages of disease progression, even before the onset of overt bulbar symptoms. The AAC evaluation should, therefore, be recommended at the time of diagnosis, regardless of whether speech impairment exists. The initial screening conducted in the clinic can introduce the AAC concepts to patients and families early in the disease course, with the implementation of 


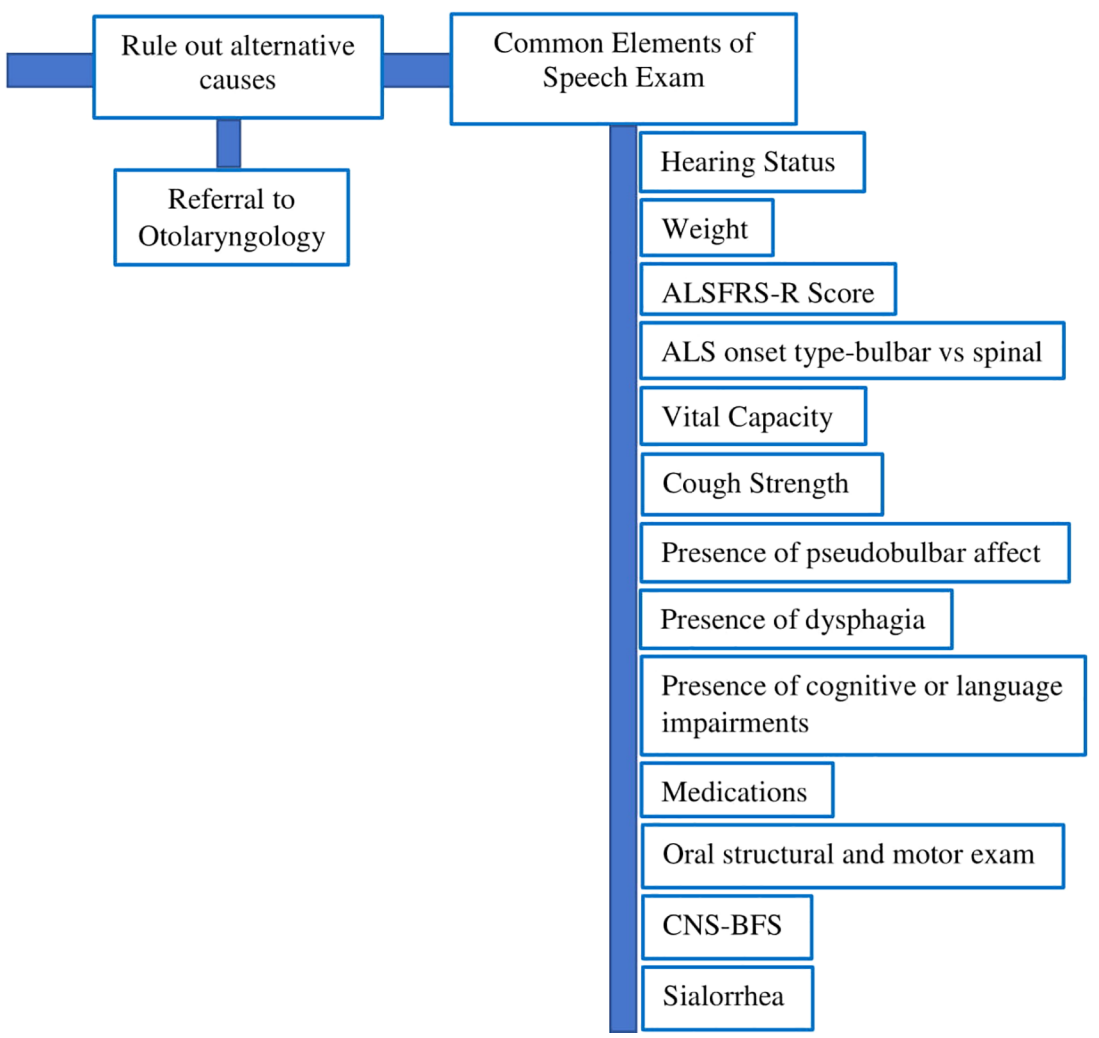

FIGURE 1. Speech evaluation. The common data elements suggested for inclusion in all clinical evaluations. ALSFRS-R, ALS Functional Rating Scale-Revised; CNS-BFS, Center for Neurological Study-Bulbar Function Scale.

technology options at a later date, as appropriate (Fig. 3). Patient and family counseling regarding the broader definition of augmentative communication should also be initiated early, emphasizing the focus upon broad support of communication through speaking, writing, texting and/or computer interfacing in the event of ineffective speech.

Guidance regarding $A A C$ referrals. The AAC section agreed that the initial clinic visit was identified as the time to arrange referral to an SLP for an AAC evaluation, which is intended to be an ongoing, dynamic collaboration between the patient, physician, and SLP, and not limited to specific technology selection. The purpose of each evaluation is to identify and implement strategies to preserve communication, and to compensate for reduced ability to communicate effectively.

Swallowing Section: Clinical Swallow Evaluation. The group initially identified existing barriers or challenges within ALS centers, including: (1) timing of referral to SLP services, (2) inconsistencies in the assessment and management practices for dysphagia, and (3) specific timing of subsequent swallow interventions. It was recommended that the SLP clinical swallowing screen should include objective testing in each of the following 5 domains: (1) patientreported outcomes; (2) dietary intake; (3) pulmonary function and airway defense physiologic capacity; (4) bulbar function; and (5) a dysphagia/aspiration screen.

Guidance regarding Swallowing referrals. SLP clinical swallow referrals should be established early in the disease course. Table 1 provides an overview of the importance in gathering clinical information across each of the above domains, as well as the supporting evidence within the ALS population for specific assessment tools. Objective measures allow for documentation of disease progression on swallowing, including dysphagia-related symptoms, impact on quality-of-life and dietary intake. The estimated time and cost of each of these proposed assessments is as follows: the Eating Assessment Tool-10 is free of charge and can be administered to the patient while in the waiting room, the Iowa Oral Pressure Instrument takes 4 min to perform, the cough peak flow (CPF) takes roughly 1 min with a device cost of approximately $\$ 45$, and the Yale Swallow Protocol is free of charge, requiring $1 \mathrm{~min}$ to complete. Objective measures of voluntary cough airflow have been noted to demonstrate significant discriminant ability to detect ALS patients at risk for penetration/ aspiration, ${ }^{1}$ and have been suggested as a useful index of airway defense physiologic capacity for inclusion in the clinical swallowing evaluation by the SLP. ${ }^{11,12}$ 


\section{BULBAR CASE HISTORY INFORMATION SPEECH EVALUATION}

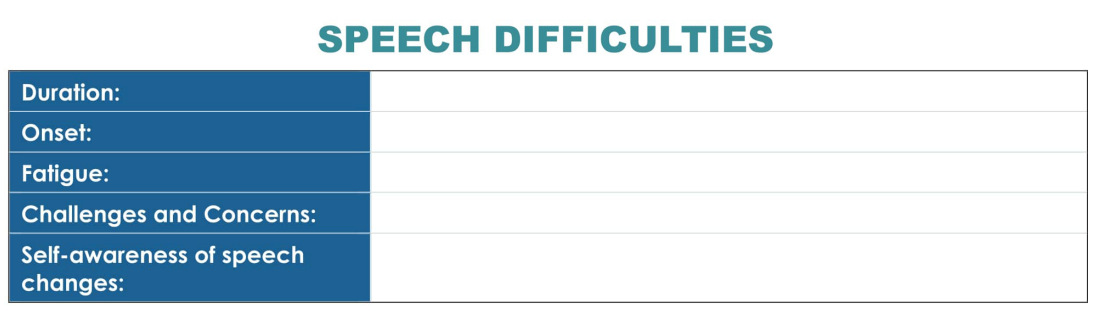

CLINICAL SPEECH ASSESSMENT

\begin{tabular}{|c|c|c|}
\hline 1. & $\begin{array}{l}\text { Patient reported } \\
\text { outcome measure (e.g. } \\
\text { CNS-BFS): }\end{array}$ & \\
\hline 2. & $\begin{array}{l}\text { Family or caregiver } \\
\text { interview: }\end{array}$ & \\
\hline 3. & Rate speech severity: & Normal | Questionable/Very Mild | Mild | Moderate | Severe \\
\hline 4. & Impressions of speech: & \\
\hline \multirow[t]{5}{*}{5.} & $\begin{array}{l}\text { Subsystem involvement } \\
\text { (observations) }\end{array}$ & \\
\hline & a. Respiration: & \\
\hline & b. Phonation: & \\
\hline & c. Resonance: & \\
\hline & d. Articulation: & \\
\hline & $\begin{array}{l}\text { Speaking rate based on } \\
\text { recording of } \\
\text { standardized passage } \\
\text { (e.g., Bamboo or } \\
\text { Rainbow passage): }\end{array}$ & words per minute \\
\hline & $\begin{array}{l}\text { Maximum syllable } \\
\text { repetition rate } \\
\text { ("puhtuhkuh"): }\end{array}$ & $\begin{array}{c}\text { Syllables per second } \\
\text { (articulatory) }\end{array}$ \\
\hline & $\begin{array}{l}\text { Maximum sustained } \\
\text { phonation /a/: }\end{array}$ & seconds (phonatory) \\
\hline
\end{tabular}

FIGURE 2. Bulbar case history information, speech evaluation. CNS-BFS, Center for Neurological Study-Bulbar Function Scale

Common data elements of clinical swallowing exam.

The distinction between a swallowing screen and a swallowing assessment was emphasized. The screen should incorporate a pragmatic approach in managing a triage flow of care within a busy ALS clinical setting. Specifically, it was recommended that all ALS patients undergo a swallowing (dysphagia/aspiration) screen, and those patients presenting with markers of dysfunction (e.g., failed screening measure) be routed for a comprehensive swallowing evaluation, which may also include an instrumental assessment of swallowing function (e.g., Videofluoroscopic Swallow Study, VFSS). Although the panel of experts in this section believed that an instrumental swallowing exam was important for patients demonstrating a high risk for aspiration and dysphagia, recent survey data reveal that only $27 \%$ of sites are routinely administering a VFSS. ${ }^{6}$ Given the established high rate of silent aspiration in ALS (i.e., no attempt to expectorate aspirated material), ${ }^{13}$ the panel agreed that direct visualization of swallowing function (safety and efficiency) and evaluation of the effectiveness of trialed swallowing maneuvers or strategies will most reliably be assessed through the use of instrumental techniques in this patient population. An open dialogue concerning the relative role, timing, and utility of the VFSS in the evaluation and monitoring of swallowing function in individuals with ALS should continue to evolve, with consideration given to the availability of specialized and highly trained SLPs in the ALS multidisciplinary clinic setting, in whose absence the VFSS may become an essential tool to accurately determine swallowing risk.

The inclusion of patient and caregiver education was especially stressed to address: (1) the role of feeding tubes for supplemental nutrition and hydration, (2) the importance of proper oral hygiene, emphasizing the association between poor oral hygiene and aspiration pneumonia in dysphagia, (3) compensatory swallow strategies and maneuvers, (4) dietary modifications including food texture and consistency, and (5) pulmonary hygiene with airway clearance and basic life saving techniques. 
Dear Colleague,

I am referring for a comprehensive AAC assessment,

however, during today's clinic the following were identified as immediate needs to address:
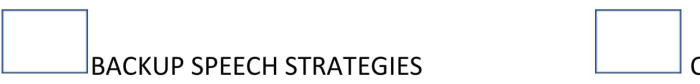
QUICK ACCESS ELECTRONIC ENCODING
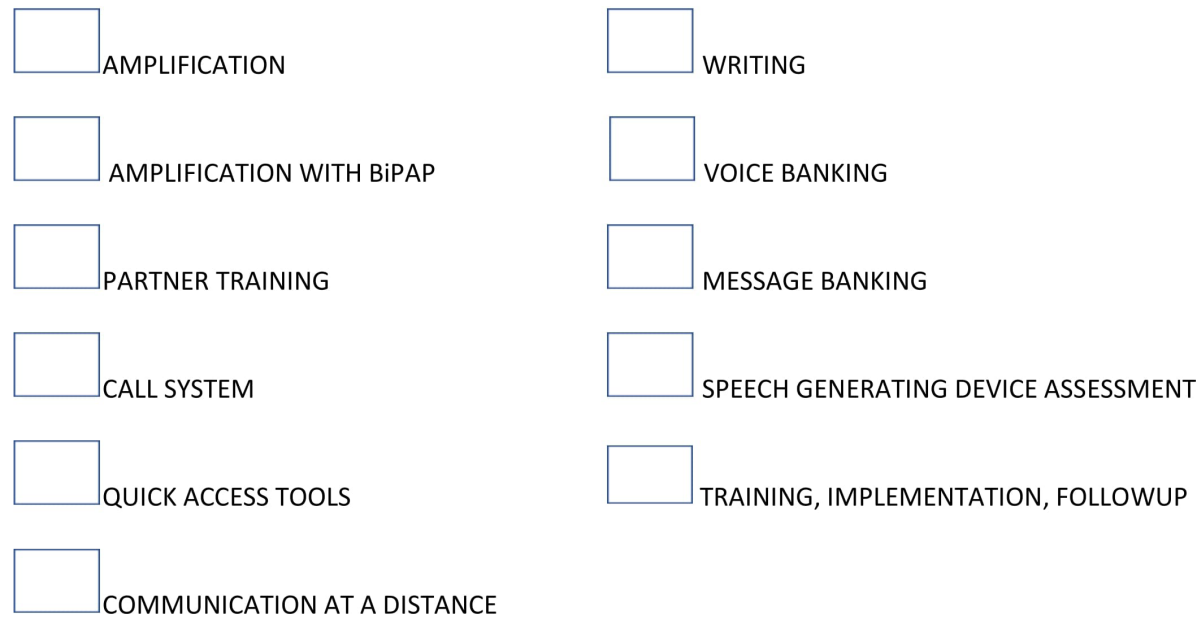

FIGURE 3. Referral goals for AAC evaluation. This triage tool should provide guidance to the speech language pathologist in the ALS clinic as to the immediate needs to be addressed in an AAC assessment for a person with ALS. It may serve as the basis for defining the referral content.

To summarize, if the patient fails a swallow screen or presents with other signs or symptoms of bulbar dysfunction (e.g., patient reported difficulties or reduced pulmonary clearance), a referral for a thorough swallowing assessment is warranted.
If an SLP is not present in the clinic or does not routinely see all scheduled clinic patients, the neurologist should initially examine each patient, focusing on the following items: the presence of swallowing difficulties, how food is being prepared, assistance with feeding,

Table 1. Clinical swallowing screen: five important assessment domains

\begin{tabular}{|c|c|c|c|}
\hline Domain & Clinical importance & Assessment tool* & Evidence available \\
\hline 1-Patient-reported outcome & $\begin{array}{l}\text { Document changes in swallowing-related } \\
\text { symptomatology during disease } \\
\text { progression }\end{array}$ & Eating Assessment Tool-10 ${ }^{15}$ & $\begin{array}{l}\text { Patients with EAT-10 scores } \geq 8 \text { were } \\
3 \text { times more likely to aspirate } \\
(95.5 \% \text { negative predictive value })^{14}\end{array}$ \\
\hline \multirow[t]{2}{*}{ 2-Dietary intake } & Rating of oral intake (current diet) & $\begin{array}{l}\text { ALS Severity Scale-Swallowing } \\
\text { Subscale }\end{array}$ & Published data ${ }^{17}$ \\
\hline & $\begin{array}{l}\text { Document diet changes during disease } \\
\text { progression }\end{array}$ & $\begin{array}{l}\text { Neuromuscular Disease Swallow } \\
\text { Status Scale (NdSSS) }\end{array}$ & Published data $^{18}$ \\
\hline \multirow[t]{3}{*}{$\begin{array}{l}\text { 3-Pulmonary function and } \\
\text { airway defense capacity }\end{array}$} & $\begin{array}{l}\text { Physiologic index of pulmonary health and } \\
\text { airway protection clearance ability }\end{array}$ & Forced vital capacity (FVC) & $\begin{array}{l}\text { FVC }<60 \% \text { predicted suggests } \\
\text { significant clinical pulmonary } \\
\text { involvement }\end{array}$ \\
\hline & $\begin{array}{l}\text { Role in decision-making for feeding tube and } \\
\text { tracheostomy tube placements }\end{array}$ & Cough testing & $\begin{array}{l}\text { Patients with peak expiratory flow } \\
(\mathrm{PEF})<4.0 \mathrm{~L} / \mathrm{s} \text { were } 3.4 \text { times more } \\
\text { likely to aspirate }{ }^{11}\end{array}$ \\
\hline & $\begin{array}{l}\text { Document pulmonary function changes } \\
\text { during disease progression }\end{array}$ & & \\
\hline \multirow[t]{3}{*}{ 4-Bulbar function } & $\begin{array}{l}\text { Anatomical and physiological integrity of } \\
\text { head and neck structures }\end{array}$ & Oral motor exam (I\&I Test) & Published data ${ }^{20}$ \\
\hline & $\begin{array}{l}\text { Document bulbar functional changes during } \\
\text { disease progression }\end{array}$ & $\begin{array}{l}\text { Lingual function (measured via the } \\
\text { lowa Oral Performance } \\
\text { Instrument - IOPI) }\end{array}$ & $\begin{array}{l}\text { Maximum tongue pressure }<21.0 \mathrm{kPa} \\
\text { was associated with swallowing }^{\text {dysfunction }}{ }^{19}\end{array}$ \\
\hline & & $\begin{array}{l}\text { Center for Neurologic Study } \\
\text { Bulbar Function Scale (CNS- } \\
\text { BFS) }\end{array}$ & Published data ${ }^{7}$ \\
\hline $\begin{array}{l}\text { 5-Dysphagia/aspiration } \\
\text { screening }\end{array}$ & $\begin{array}{l}\text { If patient fails, recommend comprehensive } \\
\text { swallow evaluation, which may include an } \\
\text { instrumental assessment of swallowing } \\
\text { physiology }\end{array}$ & Yale Swallow Protocol ${ }^{16}$ & Unpublished data \\
\hline
\end{tabular}

*Tools reported here are only those with evidence specifically examining the ALS population. Reader is advised that other validated tools may exist within a domain, although have not specifically been validated for use with the ALS population. 
unintentional weight loss, coughing or choking with meals, length of time needed to eat, saliva management, speaking rate, and slurring of speech. Should the clinical exam identify bulbar dysfunction, i.e., findings reveal tongue weakness, dysarthria with weak cough $(\mathrm{CPF}<240 \mathrm{~L} / \mathrm{min})$, and/or compromised respiratory support (FVC $<60 \%$ predicted), then an SLP referral is warranted for further comprehensive assessment. The addition of a set of minimally invasive, quick and sensitive (high yield) objective swallowing metrics were recommended, as presented in Table 1. This highlights the NEALS Bulbar recommendations for consideration of 5 important domains related to swallowing function, and the current suggestions for objective, validated tests with the inclusion of associated outcomes.

\section{DISCUSSION}

The primary intent of this project was to develop and establish the currently recognized and optimal bulbar ALS practice parameters, representing an expert-based standard of care for immediate implementation within the ALS clinical setting. Ultimately, through the application of methodological techniques incorporating available systematic online reviews of each of the summary recommendations, a formal set of bulbar ALS guidelines will be established. These formal guidelines would subsequently require the inclusion of patient and caregiver participation, and undergo future modifications as new evidence-based, clinical bulbar assessment strategies and applications are identified.

A recognized limitation of this project is the lack of existing evidence-based support for the currently proposed bulbar measures, a critical direction for future research. The inclusion of cognitive and behavioral assessment scales are also omitted due to the time constraints within a busy ALS clinic, and the lack of a universally accepted and validated cognitive scale which adequately assesses patients with bulbar dysfunction. The time required to complete these proposed bulbar assessments may also represent a limitation, yet the estimated combined time for completion of speech and swallowing assessments should be less than $20 \mathrm{~min}$. The limited existing and validated objective swallow measures identified to date highlights the critical need to pursue ongoing clinical research, focusing on the assessment of bulbar dysfunction as it relates to swallowing dynamics and its impact on clinical management.

In conclusion, the purpose of this working group project is to create a best practice set of bulbar assessment guidelines, designed for clinical implementation throughout the broader ALS community. This goal should most effectively be accomplished through the establishment of a productive collaboration between international ALS researchers, clinicians, patients, and caregivers, specifically focusing on motor speech, swallow, and communication assessment. This proposed ongoing "bulbar dialogue" should hasten the creation of an evidence-based and validated set of formal bulbar ALS guidelines, attainable within the foreseeable future. The authors extend their gratitude to Cytokinetics and the Center for Neurological Studies (CNS) for their generous financial support, and to NEALS for their organizational assistance.

Ethical Publication Statement: We confirm that we have read the Journal's position on issues involved in ethical publication and affirm that this report is consistent with those guidelines.

\section{REFERENCES}

1. Green JR, Yunusova Y, Kuruvilla MS, Wang J, Pattee GL, Synhorst L, et al. Bulbar and Speech Motor Assessment in ALS: Challenges and Future Directions. Amyotroph Lateral Scler Frontotemporal Degener 2013; 14:494-500.

2. Yunusova Y, Green J, Lindstrom M, Ball L, Pattee G, Zinman L. Kinematics of Disease Progression in Bulbar ALS. Commun Discord. 2010;43:6.

3. Ball LJ, Beukelman DR, Pattee GL. Timing of Speech Deterioration in People with Amyotrophic Lateral Sclerosis. J Med Speech-Lang Pathol. 2002;10:231-235.

4. Yorkston KM, Strand E, Miller R, Hillel A, Smith K. Speech Deterioration in Amyotrophic Lateral Sclerosis: Implications for the Timing of Intervention. J Med Speech-Lang Pathol 1993;1:35-46.

5. Yunusova Y, Green JR, Wang J, Pattee G, Zinman L. A Protocol for Comprehensive Assessment of Bulbar Dysfunction in Amyotrophic Lateral Sclerosis (ALS). J Vis Exp 2011;e2422.

6. Plowman EK, Tabor, LC, Wymer J, Pattee G. The Evaluation of Bulbar Dysfunction in Amyotrophic Lateral Sclerosis: Survey of Clinical Practice Patterns in the United States. Amyotroph Lateral Scler Frontotemporal Degener 2017;10:351-357.

7. Smith, R., Macklin, E.A., Myers, K., Pattee, G.L., Goslin KL, Meekins GD, et al. Assessment of Bulbar Function in Amyotrophic Lateral Sclerosis: Validation of a Self-Report Scale (CNS-BFS). Eur J Neurol 2018;25:907-e66.

8. Smith, R., Pioro, E., Myers, K., Sirdofsky M, Goslin K, Meekins G, et al. Enhanced Bulbar Function in Amyotrophic Lateral Sclerosis: The Nuedexta Treatment Trial. Neurotherapeutics 2017;14:762-772.

9. Green, J., Beukelman, D., Ball LJ. Algorithmic Estimation of Pauses in Extended Speech Samples of Dysarthric and Typical Speech. J Med Speech Lang Path 2004;12:149-154.

10. Fairbanks, G. Voice and Articulation Drill Book, $2^{\text {nd }}$ ed. New York: Harper \& Row. 1960; p 124-139.

11. Plowman EK, Watts SA, Robison R, Tabor L, Dion C, Gaziano J, et al. Voluntary Cough Airflow Differentiates Safe versus Unsafe Swallowing in Amyotrophic Lateral Sclerosis. Dysphagia 2016;3:383-390.

12. Watts, S.A., Tabor, L., Plowman, E.K. To Cough or Not to Cough? Examining the Potential Utility of Cough Testing in the Clinical Evaluation of Swallowing. Curr Phys Med Rehabil Rep 2016;4:262-276.

13. Gaziano, J., Tabor, L., Watts, S.A., Plowman, E.K. Prevalence, Timing and Source of Aspiration in Individuals with ALS. In: Proceedings of the 23rd Annual Meeting of the Dysphagia Research Society, Chicago, IL, 2015.

14. Plowman EK, Tabor L, Robison R, Gaziano J, Dion C, Watts SA, et al. Discriminant Ability of the Eating Assessment Tool-10 to Detect Aspiration in Individuals with Amyotrophic Lateral Sclerosis. Neurogastroenterol Motil 2016;28:85-90.

15. Belafsky PC, Mouadeb DA, Rees CJ, Pryor JC, Postma GN, Allen J, et al. Validity and reliability of the Eating Assessment Tool (EAT-10). Ann Otol Rhinol Laryngol 2008; 117: 919-924.

16. Leder SB, Suiter DM. The Yale Swallow Protocol: an evidence-based approach to decision making. New York: Springer International Publishing; 2014.

17. Hillel AD, Miller RM, Yorkston K, McDonald E, Norris FH, Konikow N. Amyotrophic lateral sclerosis severity scale. Neuroepidemiology 1989; 8:142-150.

18. Wada A, Kawakami M, Liu M, Otaka E, Nishimura A, Liu F, et al. Development of a new scale for dysphagia in patients with progressive neuromuscular diseases: the Neuromuscular Disease Swallowing Status Scale (NdSSS). J Neurol 2015; 262: 2225-2231.

19. Hiraoka A, Yoshikawa M, Nakamori M, Hosomi N, Nagasaki T, Mori T, et al. Maximum tongue pressure is associated with swallowing dysfunction in ALS patients. Dysphagia 2017; 32: 542-547.

20. Koch I, Ferrazzi A, Busatto C, Ventura L, Palmer K, Stritoni P, et al. Cranial nerve examination for neurogenic dysphagia patients. Otolaryngol 2017;7:4. 